Supporting Information

\title{
Novel C-Ring-Hydroxy-substituted Controlled Deactivation Cannabinergic analogues
}

Shashank Kulkarni, ${ }^{\dagger}$ Spyros P. Nikas, ${ }^{*}{ }^{\dagger}$ Rishi Sharma, ${ }^{\dagger}$ Shan Jiang, ${ }^{\dagger}$ Carol A. Paronis,${ }^{\dagger}{ }^{\ddagger}$ Michael Z. Leonard, ${ }^{\dagger}$ Bin Zhang, ${ }^{\dagger}$ Chandrashekhar Honrao, ${ }^{\dagger}$ Srikrishnan Mallipeddi, ${ }^{\dagger}$ Jimit Girish Raghav, ${ }^{\dagger}$ Othman Benchama, ${ }^{\dagger}$ Torbjörn U.C. Järbe, ${ }^{\dagger}$ Jack Bergman, ${ }^{\dagger}$ and Alexandros Makriyannis. ${ }^{*},{ }^{\dagger}, \#$

${ }^{\dagger}$ Center for Drug Discovery and Departments of Chemistry and Chemical Biology and Pharmaceutical Sciences, Northeastern University, Boston, Massachusetts 02115, United States

${ }^{\dagger}$ McLean Hospital, Harvard Medical School, Belmont, Massachusetts 02478, United States

${ }^{\#}$ King Abdulaziz University, Jeddah, 22254, Saudi Arabia

*To whom correspondence should be addressed.

\section{List of Contents}

- Elemental analyses (Table 1S) ....................................... Page S2.

- Time course of hypothermic effects for all tested doses of $\mathbf{3 a}, \mathbf{3 b}, \mathbf{3} \mathbf{c}$, and $11-\mathrm{OH}-\Delta^{8}-\mathrm{THC}$ -

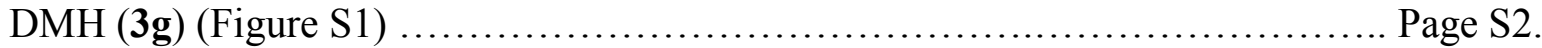

- Chemical structure of compound 28 (Figure S2) .................................. Page S3. 
Table 1S: Elemental analyses

\begin{tabular}{llll}
\hline Compd. & Mol. Formula & Calcd. & Found \\
\hline 3a & $\mathrm{C}_{24} \mathrm{H}_{34} \mathrm{O}_{5}$ & C: $71.61 ; \mathrm{H}: 8.51$ & C: $71.89 ; \mathrm{H}: 8.19$ \\
$\mathbf{1 6}$ & $\mathrm{C}_{23} \mathrm{H}_{32} \mathrm{O}_{5}$ & C: $71.11 ; \mathrm{H}: 8.30$ & C: $70.94 ; \mathrm{H}: 8.02$
\end{tabular}

3a
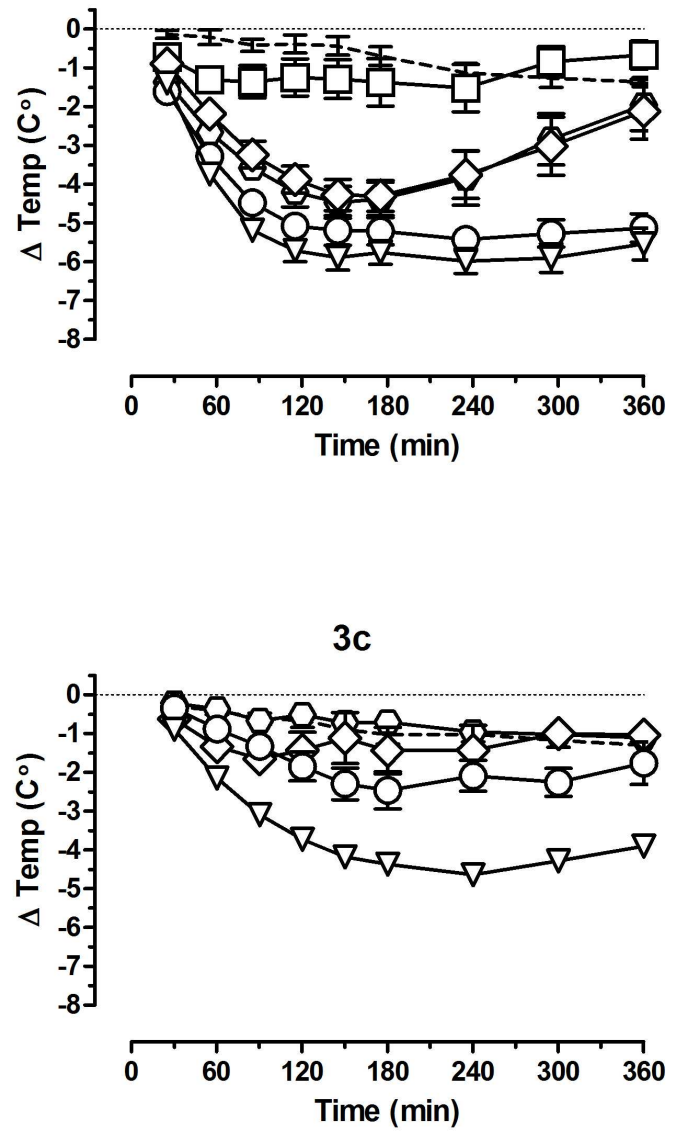

3b

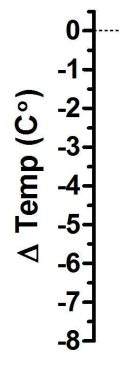

\begin{tabular}{|ll|}
\hline.-- & Vehicle \\
$\Delta$ & $0.01 \mathrm{mg} / \mathrm{kg}$ \\
$\square$ & $0.03 \mathrm{mg} / \mathrm{kg}$ \\
$\diamond$ & $0.1 \mathrm{mg} / \mathrm{kg}$ \\
$\diamond$ & $0.3 \mathrm{mg} / \mathrm{kg}$ \\
\hdashline & $1.0 \mathrm{mg} / \mathrm{kg}$ \\
$\nabla$ & $3.0 \mathrm{mg} / \mathrm{kg}$ \\
\hline
\end{tabular}
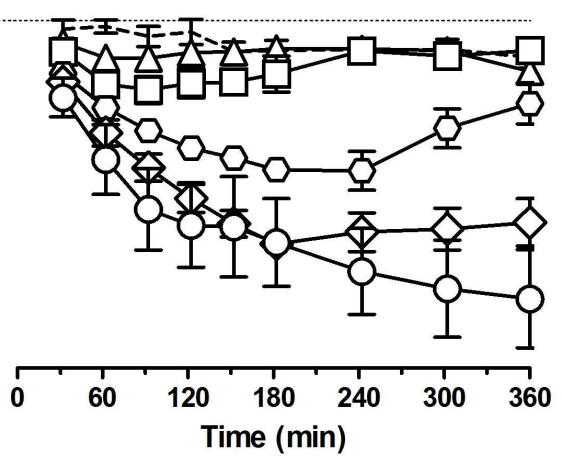

Time (min)

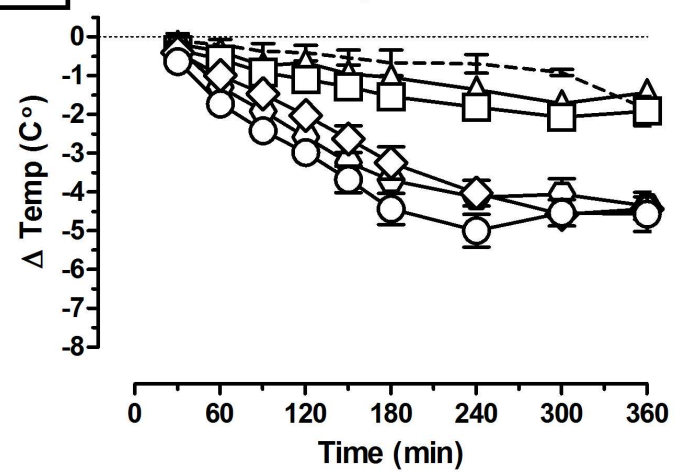

Figure S1. Time course of hypothermic effects for all tested doses of $\mathbf{3 a}, \mathbf{3 b}, \mathbf{3} \mathbf{c}$, and 11-OH- $\Delta^{8}-$ THC-DMH (3g) using female Sprague-Dawley rats $(\mathrm{n}=6)$. Abscissa: time (in min) after injection; ordinate; change in rectal body temperature. 


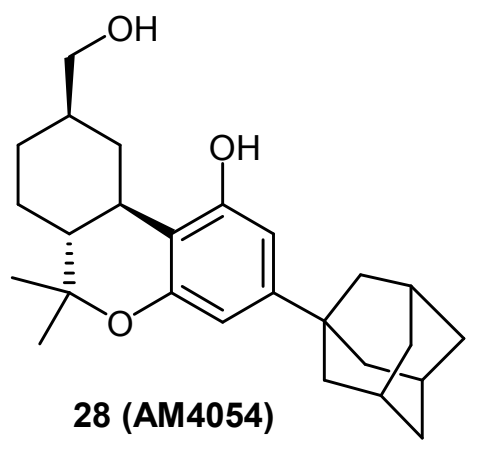

Figure S2. Chemical structure of $(6 \mathrm{a} R, 9 R, 10 \mathrm{a} R)-3$-(adamantan-1-yl)-9-(hydroxymethyl)-6, 6dimethyl-6a,7,8,9,10,10a-hexahydro-6H-benzo[c]chromen-1-ol (28, AM4054). 